\title{
STRICTLY POSITIVE DEFINITE FUNCTIONS ON SPHERES
}

\author{
YUAN XU AND E. W. CHENEY
}

(Communicated by Palle E. T. Jorgensen)

\begin{abstract}
A sufficient condition is given for the strict positive-definiteness of a real, continuous function on the $m$-dimensional sphere.
\end{abstract}

\section{INTRODUCTION}

We denote by $S^{m}$ the unit sphere in the Euclidean space $\mathbb{R}^{m+1}$. The usual geodesic distance on $S^{m}$ is denoted by $d_{m}$. Thus,

$$
d_{m}(x, y)=\operatorname{Arccos}\langle x, y\rangle \text {. }
$$

A continuous function $g:[0, \pi] \rightarrow \mathbb{R}$ is said to be positive definite on $S^{m}$ if, for any $n \in \mathbb{N}$ and for any set of $n$ points $x_{1}, x_{2}, \ldots, x_{n}$ in $S^{m}$, the $n \times n$ matrix $A$ having elements $A_{i j}=g\left(d_{m}\left(x_{i}, x_{j}\right)\right)$ is nonnegative definite:

$$
c^{T} A c=\sum_{i=1}^{n} \sum_{j=1}^{n} c_{i} c_{j} g\left(d_{m}\left(x_{i}, x_{j}\right)\right) \geq 0, \quad c=\left(c_{1}, \ldots, c_{n}\right) \in \mathbb{R}^{n} .
$$

In the celebrated paper [S], Schoenberg characterized the positive definite functions on $S^{m}$ as those functions of the form

$$
g(t)=\sum_{k=0}^{\infty} a_{k} P_{k}^{(\lambda)}(\cos t)
$$

in which $\lambda=(m-1) / 2, a_{k} \geq 0$, and $\sum a_{k} P_{k}^{(\lambda)}(1)<\infty$. Here $P_{k}^{(\lambda)}$ denotes the standard Gegenbauer ("ultraspherical") polynomial [Sz, p. 81].

If the matrices $A$ in the previous definition are positive definite for all $n$ and for all sets of $n$ distinct points $x_{1}, x_{2}, \ldots, x_{n}$ in $S^{m}$, we say that $g$ is strictly positive definite on $S^{m}$. In this note we give sufficient conditions for $g$ to be strictly positive definite.

Our interest in strictly positive definite functions is motivated by the problem of interpolating scattered data on a sphere. Suppose that numerical values $\lambda_{1}, \lambda_{2}, \ldots, \lambda_{n}$ are associated with certain prescribed points $x_{1}, x_{2}, \ldots, x_{n}$ on $S^{m}$. If a strictly positive definite function $g$ is available, then it is possible

Received by the editors February 18, 1991.

1991 Mathematics Subject Classification. Primary 43A35, 43A90, 42A82, 41 A05. 
to interpolate the data by a function of the form

$$
F(x)=\sum_{j=1}^{n} c_{j} g\left(d_{m}\left(x, x_{j}\right)\right), \quad x \in S^{m} .
$$

Indeed, the interpolation conditions become

$$
\lambda_{i}=F\left(x_{i}\right)=\sum_{j=1}^{n} c_{j} g\left(d_{m}\left(x_{i}, x_{j}\right)\right) \quad(1 \leq i \leq n) .
$$

The coefficient matrix of this system of equations is symmetric and positive definite; hence the numerical inversion is stable and can be effected by a variety of well-understood methods, including iterative procedures and the Cholesky factorization. Some work on this type of interpolation (even with functions $g$ that are not strictly positive definite) has been reported in [LC].

First, we treat the case $m=1$, as our result in this case is slightly stronger and its proof is a model for the general case. As in [S], $P_{k}^{(0)}$ can be taken to be $T_{k}$ (the $k$ th Chebycheff polynomial). Thus, $P_{k}^{(0)}(\cos \theta)=\cos k \theta$.

Theorem 1. Let $g(t)=\sum_{k=0}^{\infty} a_{k} \cos k t$, where $a_{k} \geq 0$ for all $k$ and $\sum_{k=0}^{\infty} a_{k}<$ $\infty$. Let $x_{1}, \ldots, x_{n}$ be distinct points on $S^{1}$. In order that the matrix $A_{i j}=$ $g\left(d_{1}\left(x_{i}, x_{j}\right)\right)$ be positive definite, it is sufficient that the coefficients $a_{k}$ be positive for $0 \leq k \leq[n / 2]$.

Proof. By Schoenberg's Theorem, $A$ is nonnegative definite. To prove that it is positive definite under the given hypotheses, we assume the condition $c^{T} A c=0$ and prove that $c=0$. Write $x_{i}=\left(\cos \theta_{i}, \sin \theta_{i}\right)$ so that

$$
\begin{aligned}
A_{i j} & =\sum_{k=0}^{\infty} a_{k} \cos \left(k d_{1}\left(x_{i}, x_{j}\right)\right)=\sum_{k=0}^{\infty} a_{k} \cos \left(k\left(\theta_{i}-\theta_{j}\right)\right) \\
& =\sum_{k=0}^{\infty} a_{k} \cos k \theta_{i} \cos k \theta_{j}+\sum_{k=0}^{\infty} a_{k} \sin k \theta_{i} \sin k \theta_{j} .
\end{aligned}
$$

This equation indicates that $A=E+F$, where

$$
E_{i j}=\sum_{k=0}^{\infty} a_{k} \cos k \theta_{i} \cos k \theta_{j}, \quad F_{i j}=\sum_{k=0}^{\infty} a_{k} \sin k \theta_{i} \sin k \theta_{j} .
$$

Both $E$ and $F$ are sums of nonnegative definite matrices (of rank 1) and are therefore nonnegative definite. It follows from the assumption $c^{T} A c=0$ that $c^{T} E c=c^{T} F c=0$. Thus, we have

$$
\begin{aligned}
0 & =\sum_{i=1}^{n} \sum_{j=1}^{n} c_{i} c_{j} E_{i j}=\sum_{k=0}^{\infty} a_{k} \sum_{i=1}^{n} c_{i} \cos k \theta_{i} \sum_{j=1}^{n} c_{j} \cos k \theta_{j} \\
& =\sum_{k=0}^{\infty} a_{k}\left(\sum_{i=1}^{n} c_{i} \cos k \theta_{i}\right)^{2} .
\end{aligned}
$$

Let $r=[n / 2]$. Since the coefficients $a_{0}, a_{1}, \ldots, a_{r}$ are positive by hypothesis, we see that $\sum_{i=1}^{n} c_{i} \cos k \theta_{i}=0$ for $0 \leq k \leq r$. Hence, the linear functional $\mathscr{L}$ defined by

$$
\mathscr{L}(f)=\sum_{i=1}^{n} c_{i} f\left(\theta_{i}\right) \quad\left(f \in C_{2 \pi}\right)
$$


annihilates the functions $1, \cos \theta, \cos 2 \theta, \ldots, \cos r \theta$. The same argument shows that $\mathscr{L}$ annihilates $\sin \theta, \sin 2 \theta, \ldots, \sin r \theta$. By the interpolating properties of trigonometric polynomials, there is a trigonometric polynomial, $p$, of degree at most $r$ such that $p\left(\theta_{i}\right)=c_{i}$ for $1 \leq i \leq n$. (This requires $2 r+1 \geq n$.) Hence

$$
0=\mathscr{L}(p)=\sum_{i=1}^{n} c_{i} p\left(\theta_{i}\right)=\sum_{i=1}^{n} c_{i}^{2}
$$

Theorem 2. Let $m$ be a positive integer. Set $\lambda=(m-1) / 2$. Let

$$
g(t)=\sum_{k=0}^{\infty} a_{k} P_{k}^{(\lambda)}(\cos t), \quad a_{k} \geq 0, \quad \sum_{k=0}^{\infty} a_{k} P_{k}^{(\lambda)}(1)<\infty .
$$

Let $x_{1}, x_{2}, \ldots, x_{n}$ be $n$ distinct points on $S^{m}$. In order that the $n \times n$ matrix $A_{i j}=g\left(d_{m}\left(x_{i}, x_{j}\right)\right)$ be positive definite it is sufficient that the coefficients $a_{k}$ be positive for $0 \leq k<n$.

Proof. The case $m=1$ is in Theorem 1. Assume now that $m \geq 2$. We adopt the polar coordinate system of [S], employing angular variables $\theta, \phi_{1}, \phi_{2}, \ldots$, $\phi_{m}$. The polar system depends upon a point $p$ (the "pole"), which we choose in such a way that the inner products $\left\langle p, x_{i}\right\rangle$ form a set of $n$ distinct numbers in the open interval $(-1,1)$. It is elementary to prove that such a point exists. Each point $x_{i}$ has a representation in the form

$$
x_{i}=\left(\cos \theta_{i},\left(\sin \theta_{i}\right) y_{i}\right), \quad y_{i} \in S^{m-1}, \cos \theta_{i}=\left\langle p, x_{i}\right\rangle .
$$

(One can interpret $y_{i}$ as the curvilinear projection of $x_{i}$ onto the "equator" of $S^{m}$, which is $S^{m-1}$.) An easy calculation establishes that

$$
\cos \left(d_{m}\left(x_{i}, x_{j}\right)\right)=\cos \theta_{i} \cos \theta_{j}+\sin \theta_{i} \sin \theta_{j} \cos \left(d_{m-1}\left(y_{i}, y_{j}\right)\right) .
$$

Except for variations in notation, this is equation (2.10) of [S]. Next we require the addition formula for Gegenbauer polynomials [A, p. 30]:

$$
\begin{aligned}
& P_{k}^{(\lambda)}(\cos \theta \cos \phi+\sin \theta \sin \phi \cos \psi) \\
& \quad=P_{k}^{(\lambda)}(\cos \theta) P_{k}^{(\lambda)}(\cos \phi)+\sum_{s=1}^{k} b(k, \lambda, s) Q_{k}^{(s)}(\theta) Q_{k}^{(s)}(\phi) P_{s}^{(\lambda-1 / 2)}(\cos \psi)
\end{aligned}
$$

in which we have introduced the functions

$$
Q_{k}^{(s)}(\theta)=(\sin \theta)^{s} P_{k-s}^{(\lambda+s)}(\cos \theta) \quad(0 \leq s \leq k) .
$$

(The parameter $\lambda$ has been fixed.) In equation (4), the coefficients $b(k, \lambda, s)$ are known to be nonnegative. By combining equations (3) and (4) we obtain

$$
\begin{aligned}
P_{k}^{(\lambda)}\left(\cos \left(d_{m}\left(x_{i}, x_{j}\right)\right)\right) & \\
= & P_{k}^{(\lambda)}\left(\cos \theta_{i}\right) P_{k}^{(\lambda)}\left(\cos \theta_{j}\right) \\
& \quad+\sum_{s=1}^{k} b(k, \lambda, s) Q_{k}^{(s)}\left(\theta_{i}\right) Q_{k}^{(s)}\left(\theta_{j}\right) P_{s}^{(\lambda-1 / 2)}\left(\cos \left(d_{m-1}\left(y_{i}, y_{j}\right)\right)\right) .
\end{aligned}
$$


An equation like this appears (with a typographical error) in [S, p. 101]. From the above equations, we obtain

$$
\begin{aligned}
A_{i j}= & \sum_{k=0}^{\infty} a_{k} P_{k}^{(\lambda)}\left(\cos \theta_{i}\right) P_{k}^{(\lambda)}\left(\cos \theta_{j}\right) \\
& +\sum_{k=0}^{\infty} a_{k} \sum_{s=1}^{k} b(k, \lambda, s) Q_{k}^{(s)}\left(\theta_{i}\right) Q_{k}^{(s)}\left(\theta_{j}\right) P_{s}^{(\lambda-1 / 2)}\left(\cos \left(d_{m-1}\left(y_{i}, y_{j}\right)\right)\right) .
\end{aligned}
$$

As in the previous proof, we write $A=E+F$, where now

$$
\begin{aligned}
E_{i j} & =\sum_{k=0}^{\infty} a_{k} P_{k}^{(\lambda)}\left(\cos \theta_{i}\right) P_{k}^{(\lambda)}\left(\cos \theta_{j}\right), \\
F_{i j} & =\sum_{k=0}^{\infty} a_{k} \sum_{s=1}^{k} b(k, \lambda, s) Q_{k}^{(s)}\left(\theta_{i}\right) Q_{k}^{(s)}\left(\theta_{j}\right) B_{i j}^{(s)}, \\
B_{i j}^{(s)} & =P_{s}^{(\lambda-1 / 2)}\left(\cos \left(d_{m-1}\left(y_{i}, y_{j}\right)\right)\right) .
\end{aligned}
$$

The matrices $B^{(s)}$ are nonnegative definite by Schoenberg's Theorem, applied in $S^{m-1}$. The summands in the equation for $F$ are nonnegative definite because (except for the coefficients) they can be written as $D^{(s, k)} B^{(s)} D^{(s, k)}$, with

$$
D^{(s, k)}=\operatorname{diag}\left(Q_{k}^{(s)}\left(\theta_{1}\right), \ldots, Q_{k}^{(s)}\left(\theta_{n}\right)\right) .
$$

These observations show that $F$ is nonnegative definite. Since $E$ is a sum of (rank-1) nonnegative definite matrices, $E$ itself is nonnegative definite. Thus, if $c^{T} A c=0$, then we may infer that $c^{T} E c=c^{T} F c=0$. If $a_{0}, \ldots, a_{n-1}$ are all positive, $E$ will be positive definite by the argument used in the proof of the preceding theorem. Here we note that the functions $P_{0}^{(\lambda)}, \ldots, P_{n-1}^{(\lambda)}$ generate the $n$-dimensional polynomial space $\Pi_{n-1}$ and that interpolation of arbitrary data at any $n$ nodes is possible. Our choice of coordinate system ensures that the points $t_{i}=\cos \theta_{i}$ are distinct. This proves that if $a_{0}, \ldots, a_{n-1}$ are positive, $A$ is positive definite.

We do not know whether Theorem 2 is true under the weaker hypothesis that $a_{k}>0$ for $0 \leq k \leq[n / 2]$.

Corollary. In order that the function $g$ in equation (2) be strictly positive definite on $S^{m}$, it is sufficient that all coefficients $a_{k}$ be positive.

It is possible that the following recurrence formula, which we recently discovered, can be useful in proving a sharper form of Theorem 2:

(7) $4\left(\begin{array}{c}\lambda+s+1 \\ 2\end{array}\right)\left[Q_{k+2}^{(s+2)}-Q_{k}^{(s+2)}\right]=\left(\begin{array}{c}2 \lambda+k+s+1 \\ 2\end{array}\right) Q_{k}^{(s)}-\left(\begin{array}{c}k-s+2 \\ 2\end{array}\right) Q_{k+2}^{(s)}$.

This formula is valid for $\lambda \geq 1 / 2$ and $0 \leq s \leq k$. It is proved by using formulas (4.7.27) and (4.7.29) in [Sz]. These give us

$$
\begin{aligned}
2 \lambda(n+\lambda)\left(1-x^{2}\right) P_{n-1}^{(\lambda+1)}(x) & =\left(\begin{array}{c}
n+2 \lambda \\
2
\end{array}\right) P_{n-1}^{(\lambda)}(x)-\left(\begin{array}{c}
n+1 \\
2
\end{array}\right) P_{n+1}^{(\lambda)}(x) \\
(n+\lambda) P_{n}^{(\lambda)}(x) & =\lambda\left[P_{n}^{(\lambda+1)}(x)-P_{n-2}^{(\lambda+1)}(x)\right]
\end{aligned}
$$


Equations (8) and (9) are valid for $\lambda \geq-1 / 2$ and $n \geq 0$. We interpret $P_{m}^{(\lambda)}$ to be 0 when $m<0$. In equation (9), replace $n$ by $n-1$ and $\lambda$ by $\lambda+1$ to obtain an expression for $(n+\lambda) P_{n-1}^{(\lambda+1)}(x)$ that can be substituted in equation (8). After that, put $x=\cos \theta, 1-x^{2}=\sin ^{2} \theta, n=k-s+1$, and replace $\lambda$ by $\lambda+s$ to prove equation (7).

A natural question is whether the conclusions in Theorems 1 and 2 are true under the assumption that some infinite set of coefficients is positive. That the answer is "No" can be seen by letting $m=1, n=2, x_{1}=(1,0), x_{2}=(0,1)$, and $g(t)=\sum_{k=1}^{\infty} 2^{-k} \cos 4 k t$. In this case the matrix $A$ is $\left(\begin{array}{ll}1 & 1 \\ 1 & 1\end{array}\right)$ and is not positive definite.

After this paper was accepted, we found sufficient conditions for the strict positive definiteness of a function on $S^{\infty}$. The result will appear in [CX].

\section{REFERENCES}

[A] Richard Askey, Orthogonal polynomials and special functions, Regional Conf. Ser. in Appl. Math., vol. 21, SIAM, Philadelphia, PA, 1975.

[CX] E. W. Cheney and Yuan Xu, A set of research problems in approximation theory, Topics in Polynomials of One and Several Variables and Their Applications (Th. M. Rassias, H. M. Srivastava, and A. Yanushauskas, eds.), World Scientific, London (to appear).

[LC] W. A. Light and E. W. Cheney, Interpolation by periodic radial basis functions, J. Math. Anal. Appl. 168 (1992), 111-130.

[S] I. J. Schoenberg, Positive definite functions on spheres, Duke Math. J. 9 (1942), 96-108.

[Sz] Gabor Szegö, Orthogonal polynomials, Amer. Math. Soc. Colloq. Publ., vol. 23, Amer. Math. Soc., Providence, RI, 1959.

Department of Mathematics, University of Oregon, Eugene, Oregon 97403-1222

E-mail address: yuan@math.uoregon.edu

Department of Mathematics, University of Texas at Austin, Austin, TeXas 78712-1082

E-mail address: cheney@math.utexas.edu 